N. E. Piskunov, W. W. Weiss, D. F. Gray, eds.

\title{
Round Table Summary: Comparison of Different NLTE Codes and the Role of Atomic Input Data
}

\author{
Inga Kamp \\ Leiden Observatory, PO Box 9513, 2300 RA Leiden, The Netherlands
}

Sergey Korotin

Department of Astronomy, Odessa State University, Shevchenko Park, 65014 Odessa

Lyudmila Mashonkina

Astronomy Department, Kazan State University, Kremlevskaya 18, 420008 Kazan, 420008 Russia

Norbert Przybilla

Universitäts-Sternwarte München, Scheinerstr. 1, 81679 München, Germany

Slava Shimansky

Department of Astronomy, Kazan State University, Kremlevskaya 18, 420008 Kazan, Russia

\begin{abstract}
We started a comparison between different NLTE codes that calculate the statistical equilibrium in solar-type and late type stars ( $G$ - A type stars). Discrepancies between different authors analyzing the statistical equilibrium of the same element in the same atmosphere are often surprisingly large. Hence, this study was meant to nail down the origin of these differences. The preliminary results indicate that even if the atmosphere and the atomic input data is fixed, discrepancies of up to $40 \%$ in the outer atmospheric layers are still present; the main reason is the different treatment of the background opacity. Following up, we shortly discuss the completeness and accuracy of atomic data used in analyses of the kinetic equilibrium of atoms in the atmospheres of middle and late type stars.
\end{abstract}

\section{Introduction}

The IAU symposium gave an excellent opportunity to bring together different NLTE codes from the stellar atmosphere community and to compare the results for some standard calculations. By NLTE code, we mean here a computer 
program to calculate the statistical equilibrium of a model atom in a fixed planeparallel stellar atmosphere in radiative and hydrostatic equilibrium.

Given the present situation in the literature, the reason for such a comparison is obvious: different authors claim different results for the same atom/ion and the same star. Unfortunately, the possibility to reproduce previous model calculations is often limited by the amount of information on input data obtained from the literature. And colleagues, who are not performing NLTE calculations themselves, but use literature results, often wonder about the accuracy of published departure coefficients.

To improve this unsatisfactory situation and to figure out the main sources of discrepancy between the codes, we set up a small test case, where we tried to fix as many possible sources of difference as possible.

Five different codes participated in this comparison: NONLTE3 (Sakhibullin 1983), NONLTE3a (Ivanova \& Shimansky 2000), DETAIL (Giddings 1981, Butler \& Giddings 1985), the Kiel code (Steenbock \& Holweger 1984), and MULTIa (Korotin et al. 1999). In fact, three of the codes, NONLTE3, NONLTE3a, and the Kiel code, are based on the Complete Linearization (CL) algorithm of Auer \& Heasely (1976). DETAIL uses instead the Accelerated Lambda Interation (ALI) from Rybicki \& Hummer (1991), while MULTIa uses the Operator Perturbation Technique (OPT) from Scharmer (1981). Table 1 lists the five codes with their different treatment of $b-f$ and $b-b$ transitions.

The calculations are performed by Lyudmila Mashonkina (NONLTE3), Slava Shimansky (NONLTE3a), Norbert Przybilla (DETAIL), Inga Kamp (Kiel code), and Sergey Korotin (MULTIa).

Table 1. Summary of the different codes with their respective algorithm and treatment of the background opacity (added to the continuous opacity) for bound-free and bound-bound transitions: ATLAS and CD 18 (Kurucz 1979, 1992, 1993), ATMOS (Baschek et al. 1966), OPS means opacity sampling, OPMET calculates the b-f opacities using TOPbase and Auer et al. (1972) data.

\begin{tabular}{ll|ll|ll} 
Name & alg. & \multicolumn{2}{|c|}{ b-f transitions } & \multicolumn{2}{c}{ b-b transitions } \\
& & $\begin{array}{l}\text { b-f } \\
\text { opacity }\end{array}$ & $\begin{array}{l}\text { b-b } \\
\text { opacity }\end{array}$ & $\begin{array}{l}\text { b-f } \\
\text { opacity }\end{array}$ & $\begin{array}{l}\text { b-b } \\
\text { opacity }\end{array}$ \\
\hline NONLTE3 & CL & ATLAS & OPS+CD 18 & ATLAS & OPS+CD 18 \\
NONLTE3a & CL & OPMET & OPSa+CD 18 & OPMET & OPSa+CD 18 \\
DETAIL & ALI & DETAIL & ATLAS ODF's & DETAIL & ATLAS ODF's \\
Kiel code & CL & ATMOS & ATLAS ODF's & ATMOS & none \\
MULTIa & OPT & ATLAS & ATLAS ODF's & ATLAS & none
\end{tabular}

\section{The test case}

We performed the NLTE calculation in two different environments, the Sun and Vega. The model atmospheres are calculated with the ATMOS code and provided as input data for the NLTE calculations. 
The model atom used for this comparison is a simplified $\mathrm{C}$ I/C II model atom, where the level energies, line data, collisional cross sections and photoionization cross sections are prescribed, so that the basic atomic data is the same for all codes. Table 2 lists the 19 energy levels and additional information, while Table 3 summarizes all necessary line data.

Table 2. Enery levels of the carbon model atom: level energy in eV, excitation energy of parent term (if not explicitly present in model atom), mean square atomic radii in units of the Bohr radius $a_{0}^{2}$, statistical weight, level number of parent term (li), level designation.

level $\mathrm{E}[\mathrm{ev}] \mathrm{DI}[\mathrm{eV}] \quad\left(\frac{\bar{r}}{a_{0}}\right)^{2} \quad \mathrm{~g} \quad \mathrm{li}$ desig.

$\begin{array}{rrrrrrr}1 & 0.0037 & 0.0000 & 0.629 & 9.00 & 15 & \text { 2P3P } \\ 2 & 1.2637 & 0.0000 & 1.226 & 5.00 & 15 & \text { 2P1D } \\ 3 & 2.6840 & 0.0000 & 2.323 & 1.00 & 15 & \text { 2P1S } \\ 4 & 4.1826 & 0.0000 & 4.427 & 5.00 & 16 & \text { 2P5S } \\ 5 & 7.4853 & 0.0000 & 34.254 & 9.00 & 15 & \text { 3S3P } \\ 6 & 7.6848 & 0.0000 & 38.077 & 3.00 & 15 & \text { 3S1P } \\ 7 & 7.9460 & 0.0000 & 31.844 & 15.00 & 16 & \text { 2P3D } \\ 8 & 8.5371 & 0.0000 & 49.880 & 3.00 & 15 & \text { 3P1P } \\ 9 & 8.6444 & 0.0000 & 54.591 & 15.00 & 15 & \text { 3P3D } \\ 10 & 8.7711 & 0.0000 & 60.987 & 3.00 & 15 & \text { 3P3S } \\ 11 & 8.8494 & 0.0000 & 65.467 & 9.00 & 15 & \text { 3P3P } \\ 12 & 9.0026 & 0.0000 & 75.678 & 5.00 & 15 & \text { 3P1D } \\ 13 & 9.1718 & 0.0000 & 89.763 & 1.00 & 15 & \text { 3P1S } \\ 14 & 9.3304 & 0.0000 & 106.572 & 9.00 & 16 & \text { 2P3P } \\ 15 & 0.0052 & 0.0000 & 1.718 & 6.00 & 20 & \text { 2P2P } \\ 16 & 5.3358 & 6.4992 & 3.313 & 12.00 & 20 & \text { 2P4P } \\ 17 & 9.2903 & 6.4992 & 5.867 & 10.00 & 20 & \text { 2P2D } \\ 18 & 11.9637 & 6.4992 & 9.254 & 2.00 & 20 & \text { 2P2S } \\ 19 & 13.7191 & 12.690 & 13.076 & 6.00 & 20 & \text { 2P2P }\end{array}$

The line broadening parameters $\gamma_{4}, \log C_{6}$, and $\gamma_{\text {rad }}$ are calculated using the following approximations

$$
\begin{aligned}
\gamma_{4}(Z=0) & =\frac{1}{2 \pi v_{10000}}\left(\frac{h}{m_{\mathrm{e}}(Z+1)}\right)^{2} \frac{5}{3} n_{\mathrm{eff}}^{4} N_{\mathrm{e}} \\
\gamma_{4}(Z \neq 0) & =\frac{1}{2 \pi v_{10000}}\left(\frac{h}{m_{\mathrm{e}}(Z+1)}\right)^{2} 4 n_{\mathrm{eff}}^{4} N_{\mathrm{e}} \\
\gamma_{6} & =8.08 C_{6}^{0.4} v^{0.6} N_{\mathrm{H}}
\end{aligned}
$$

Here $v$ is the average relative velocity of atom and perturbing particle, $n_{\text {eff }}$ the effective quantum number, $N_{\mathrm{e}}$ and $N_{\mathrm{H}}$ the number density of electrons and hydrogen atoms respectively.

The maximum collision cross section for collisions with electrons is fixed for all optically allowed collisions to $\pi a_{0}^{2}$ and for all optically forbidden transitions 
Table 3. Lines of the carbon model atom: lower (ll) and upper level (lu) of the line transition, wavelength, transition probability $\log g f$ and the two broadening parameters $\log C_{6}$ and $\gamma_{\mathrm{rad}}$.

\begin{tabular}{rrrccr} 
ll & $\mathrm{lu}$ & $\begin{array}{r}\lambda \\
{[\AA]}\end{array}$ & $\log g f$ & $\log C_{6}$ & $\begin{array}{r}\gamma_{\mathrm{rad}} \\
{\left[10^{8} \mathrm{~s}^{-1}\right]}\end{array}$ \\
\hline & & & & \\
1 & 5 & 1657.008 & -0.310 & -30.860 & 8.085 \\
1 & 7 & 1561.438 & -0.500 & -30.892 & 9.105 \\
1 & 14 & 1329.578 & -0.730 & -30.362 & 12.558 \\
2 & 6 & 1930.905 & -0.250 & -30.820 & 5.954 \\
3 & 6 & 2478.561 & -1.070 & -30.833 & 3.614 \\
5 & 9 & 10691.250 & 0.340 & -31.078 & 0.194 \\
5 & 11 & 9094.829 & 0.150 & -30.892 & 0.268 \\
6 & 8 & 14542.500 & -0.080 & -31.315 & 0.105 \\
6 & 12 & 9405.729 & 0.290 & -30.812 & 0.251 \\
6 & 13 & 8335.149 & -0.440 & -30.673 & 0.320 \\
15 & 17 & 1335.708 & -0.333 & -31.769 & 12.443 \\
15 & 18 & 1037.018 & -0.308 & -31.510 & 20.643 \\
15 & 19 & 904.142 & 0.225 & -31.331 & 27.157
\end{tabular}

to $0.1 \pi a_{0}^{2}$. Collisional ionization cross sections are calculated by

$$
C_{\max }=\left(\frac{E_{\text {ion }}(\mathrm{H})}{E_{\text {ion }}}\right)^{2} 0.665 \xi_{i},
$$

where $\xi_{i}$ denotes the number of equivalent electrons of the respective atom/ion (Drawin 1967). For this comparison, we ignore collisions with neutral particles. The energy dependence of the respective allowed/forbidden collision cross section is taken from the work of Drawin (1967).

The complete data for the test case including the model atmospheres, atomic data and some instructions can be downloaded from the following Webpage http://www.leidenuniv.nl/ kamp/NLTE/test.html.

\section{Comparison of different NLTE codes}

The first step was to achieve agreement for the LTE level population numbers. It turns out that the Kiel code accounts for molecule formation in the atmosphere, which leads in the case of a cool star like the Sun to a decrease of atomic carbon in the atmosphere. Part of the carbon is locked in molecules like $\mathrm{CH}, \mathrm{C}_{2}, \mathrm{CN}$, and CO. Molecule formation is explicitly switched off to obtain agreement in LTE with the other codes.

A second step was to make sure, that all models are calculated on the same depth scale. It turned out, that the difference between a $\log \tau_{5000}$ and a mass scale is negligible. Hence, the results of the following sections are always plotted as a function of reference optical depth at $5000 \AA$.

In the Sun, the five different codes agree in LTE to within $3 \%$ for C I and there are still some discrepancies of the order of $10 \%$ for $\mathrm{C}$ II in the MULTIa code. 
In Vega, the agreement between four codes (NONLTE3, MULTIa, DETAIL, and Kiel) is better than $1 \%$.

\subsection{The Sun}

Fig. 1 shows the relative differences in the NLTE level population numbers for the various codes. In addition, two calculations are shown using the Kiel code with a continuum background opacity for (b-b) transitions scaled by a factor 2 up and down, respectively. The difference between these two calculations and the original Kiel calculation span roughly the range of discrepancies seen in the comparison of all the other codes. Since each code uses its own recipe to calculate the background opacity, the discrepancies of up to $40 \%$ are probably entirely due to that point. In the Sun, there is only one strong $\mathrm{Ti}$ II blend at the $\mathrm{C}_{\mathrm{I}} 10691.25 \AA$ line, causing a change in the 3P3D level. The continuum opacity for (b-f) transitions is of minor importance. A scaling here changes mainly the $\mathrm{C}$ II levels, but only to a very small extent (a factor 10 scaling leads to a maximum change in level population of 10\%). Nevertheless, the 3P3S level is strongly affected, because it is not coupled by line transitions to other $\mathrm{C}$ I levels, but only coupled to $\mathrm{C}$ II via photoionization and recombination.

In addition, one has to keep in mind that the region of interest (line formation) in mostly restricted to the layers $-2<\log \tau<0$. In these layers, the agreement between the different codes is often better than $20 \%$. Moreover, it is not the individual level population number, but the ratio of the level populations that enters the line profile calculation and a comparison of the latter is left for future work.

\subsection{Vega}

In Vega, Fig. 2 illustrates that blend lines play a crucial role for the determination of level population numbers. In fact the differences can rise up to $\sim 100 \%$ (compare NONLTE3 results: dotted line and dash-dot-dotted line); in the line forming regions, the differences amount only up to $\sim 50 \%$. This additional source of discrepancy makes the NLTE comparison look even worse than for the Sun. However, one has to keep in mind that the model atom used here is oversimplified and in a complete model atom the effect of blend lines may be reduced, because there exist a manifold of other transitions (de)populating the levels of concern.

\section{Completeness and accuracy of atomic data used in NLTE studies}

The results of the comparison are very promising, making once again clear that the atomic input data is besides the treatment of background opacity the most important ingredient for NLTE calculations.

We now concentrate on some problems of atomic data used in studies of kinetic equilibrium of neutral and ionized atoms in atmospheres of middle and late type stars. In Table 4 we list the papers where the first description of original NLTE method for a specific atom was given. In some cases the later works are based on atomic data from previous studies. We refer to them as independent studies because available atomic data is important but insufficient condition to perform NLTE calculations and to obtain reliable results. 

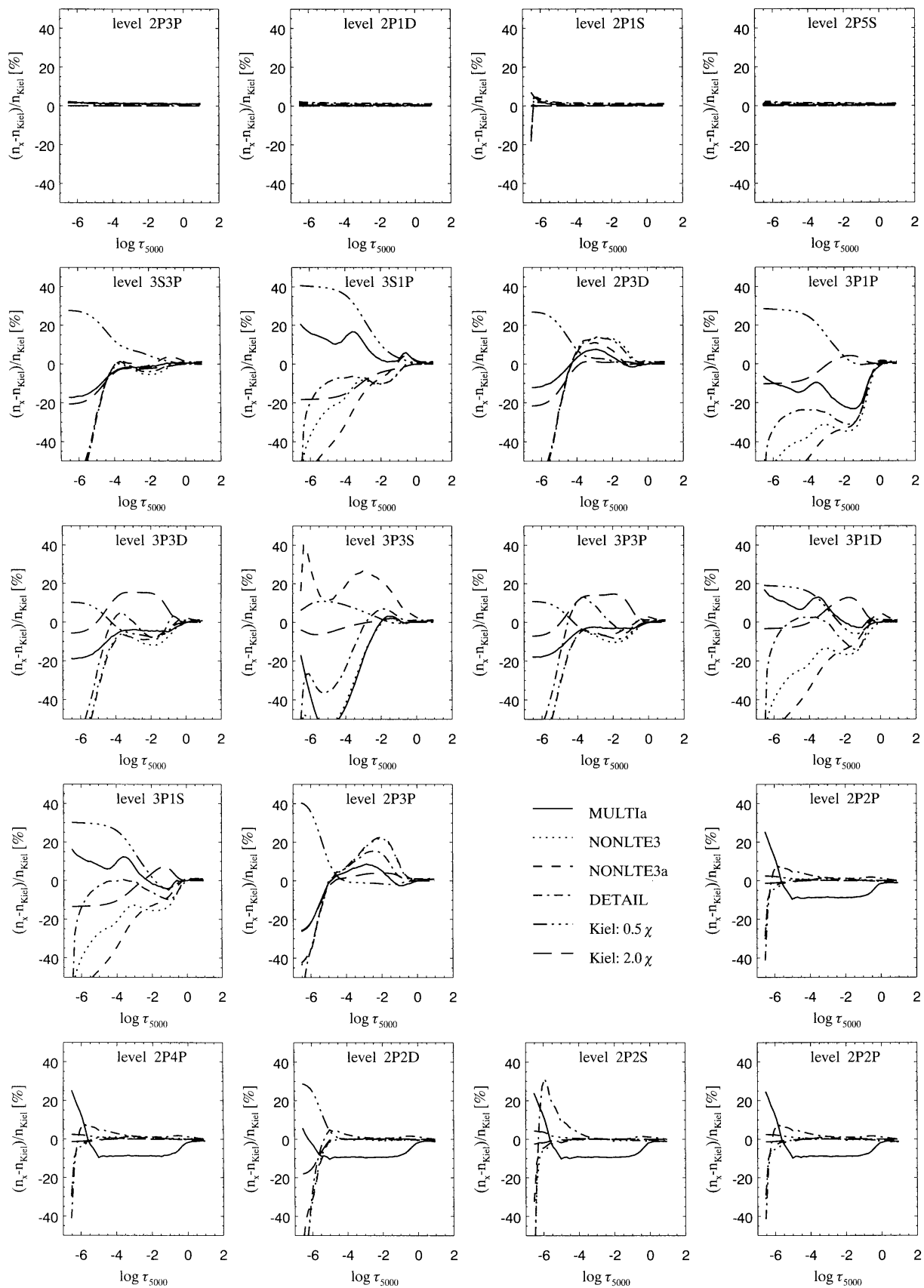

Figure 1. Deviation of NLTE level population numbers in the Sun: the results of four different codes are plotted with respect to the Kiel code in \%. In addition two more calculations with the Kiel code are plotted, where the continuum background opacity $\chi$ for all (b-b) transitions is scaled by a factor 0.5 and 2.0 respectively. 

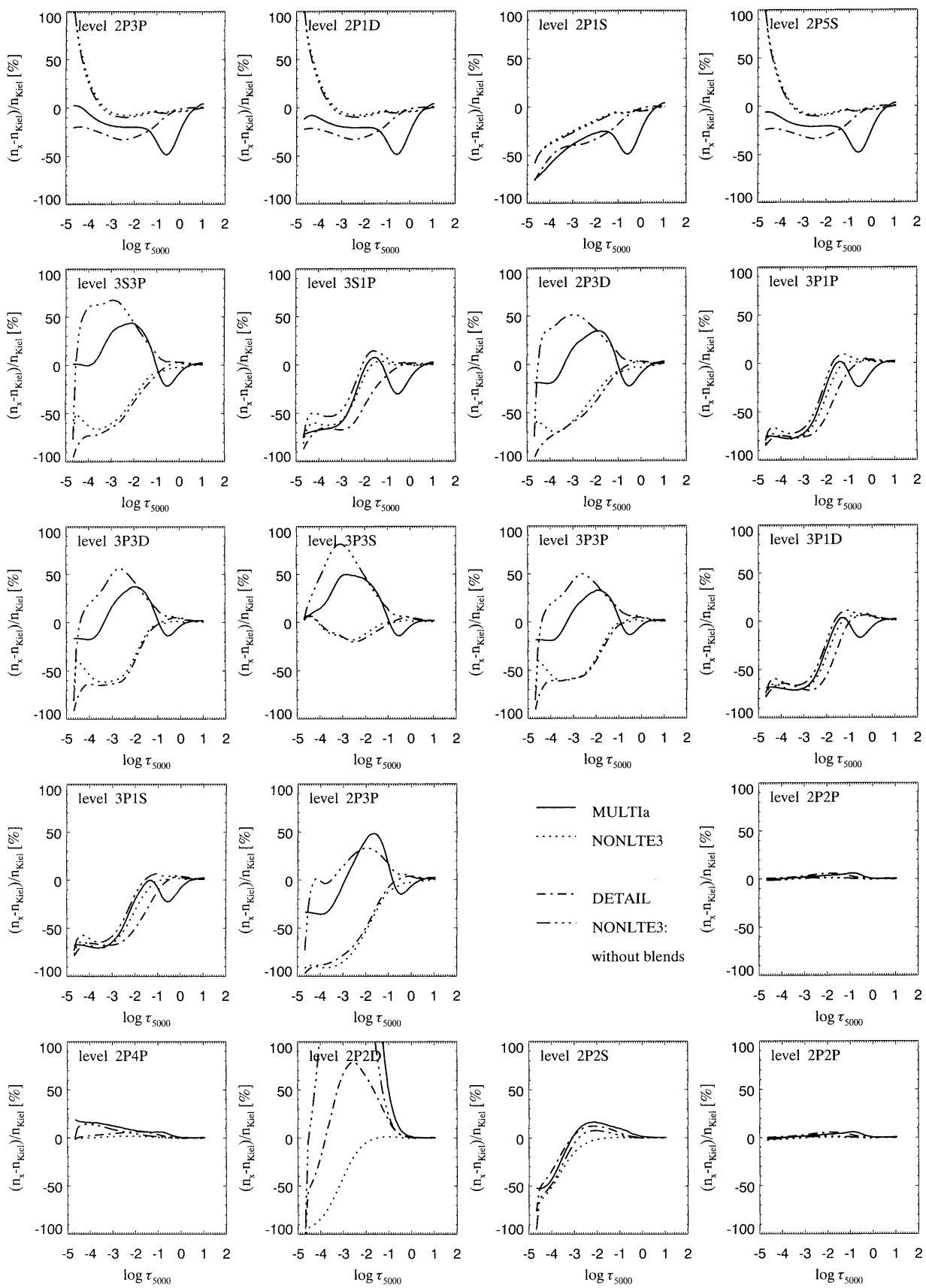

Figure 2. Deviation of NLTE level population numbers in Vega: the results of three different codes are plotted with respect to the Kiel code in \%. In addition, a calculation with the NONLTE3 code is plotted, where blends are neglected for (b-b) transitions. 
Table 4. NLTE studies for A-K stars

Li I

de La Reza \& da Silva (1981)

Steenbock \& Holweger (1984)

Pavlenko (1992)

Carlsson et al. (1994)

Kiselman \& Plez (1995)

Mashonkina et al. (2002)

B I

Kiselman (1994)

C I

Shchukina \& Shcherbina (1990)

Takeda (1992)

Rentzsch-Holm (1996)

Przybilla et al. (2001)

$\mathbf{N}_{\text {I }}$

Takeda (1992)

Rentzsch-Holm (1996)

Lemke \& Venn (1996)

Przybilla \& Butler (2001)

O I

Kiselman (1991)

Takeda (1992)

Carlsson \& Judge (1993)

Carretta \& Gratton (1994)

Reetz (1999)

Mishenina et al. (1999)

Paunzen et al. (1999)

Przybilla et al. (2000)

$\mathrm{Na}$ I

Gehren (1975)

Sakhibullin (1987)

Bruls et al. (1992)

Mashonkina et al. (1993)

Takeda (1995)

Baumüller et al. (1998)

Gratton et al. (1999)
Mg I

Gigas (1988)

Lemke \& Holweger (1987)

Carlsson et al. (1992)

Mashonkina et al. (1996)

Zhao et al. (1998)

Gratton et al. (1999)

Idiart \& Thevenin (2000)

Przybilla et al. (2001)

Al I

Baumüller \& Gehren (1996)

$\mathbf{S}_{\text {I }}$

Takada-Hidai \& Takeda (1996)

$\mathbf{K}_{\text {I }}$

Shchukina (1987)

Bruls et al. (1992)

Takeda et al. (1996)

Ivanova \& Shimansky (2000)

Ca I

Watanabe \& Steenbock (1985)

Drake (1991)

Fe I-II

Boyarchuk et al. (1985)

Steenbock (1985)

Takeda (1991)

Holweger (1996)

Shchukina et al. (1997)

Thevenin \& Idiart (1999)

Gratton et al. (1999)

Gehren et al. (2001)

Sr II

Belyakova \& Mashonkina (1997)

Ba II

Gigas (1988)

Mashonkina \& Bikmaev (1996)

Eu II

Mashonkina (2000) 
Even for cool and dense atmospheres recent studies show an advantage of using the NLTE approach compared with LTE one. Determination of Na and $\mathrm{Al}$ abundances in cool dwarfs is the prominant example. $\mathrm{Na} \mathrm{I}$ and $\mathrm{Al} \mathrm{I}$ are both minor species in atmospheres of such stars and NLTE effects are strong for both of them. Due to overionization of Al I NLTE abundance corrections for aluminium are positive and can be up to 0.7 dex (Baumüller \& Gehren 1997). In contrary, overrecombination of $\mathrm{Na}$ I results in negative NLTE abundance corrections of up to 0.5 dex by absolute value (Baumüller et al. 1998). In both cases the behaviour of the $[\mathrm{El} / \mathrm{Fe}]$ ratio with metallicity changes significantly in NLTE (Baumüller \& Gehren 1997, their Fig.7; Baumüller et al. 1998, their Fig.13) and this has important consequences for the galactic chemical evolution study. The next example concerns the fitting of solar line profiles. Using the LTE assumption, it is impossible to obtain a good fit for the observed profiles of Ba II $\lambda 6496$ (Mashonkina \& Gehren 2000, their Fig.3) and Sr II $\lambda 10327$ lines (Mashonkina \& Gehren 2001, their Fig.5) with any reasonable values of fitting parameters.

\subsection{What types of atomic data are necessary?}

In general, for each investigated atom we need data for all energy levels, their radiative and collisional ionization cross-sections, and radiative and collisional excitation cross-sections for all possible transitions among the atomic levels. However, due to missing or bad quality atomic data we reasonably restrict our model atoms and worry especially about the accuracy of that type of atomic data which has the most impact on the departures from LTE.

For minor species bound-free (b-f) transitions are mainly responsible for NLTE effects. In this case the two most important points are: 1) Accurate photoionization cross-sections for low excited levels and accurate ultraviolet opacity; 2) Close coupling to continuum should be provided by including highly excited levels (separated from ionization threshold by no larger than $\chi_{i} \sim \mathrm{kT}_{e}$ ) into the model atom.

For majority atoms the kinetic equilibrium is strongly affected by boundbound (b-b) transitions. Indeed, the most important of them are transitions between the lowest levels. In this case we should concentrate our efforts on the following two points: 1) Background opacity at line frequencies, including opacity of blending spectral lines; 2) Fine structure splitting of energy levels.

In the atmospheres of A-K stars, inelastic collisions of atoms compete with radiative processes in kinetic equilibrium, and thus collisional rates should be carefully calculated. In atmospheres of cool stars, collisions with heavy particles may be important In addition to electron impacts, since the ratio of number densities $n_{H} / n_{e}$ may exceed $10^{4}$. For majority atoms, collisional coupling of low excitation levels to the ground state and to each other should be carefully taken into account. It is worth especially to note the importance of transitions between fine structure levels.

\subsection{How accurate is atomic data?}

The recent Opacity Project and Iron Project have provided progress in the improvement of atomic data for the most abundant atoms. However, even for such atoms, there still remain some problems. 
For $\mathrm{Mg} \mathrm{I}$ and $\mathrm{Fe} \mathrm{I}$ the recent calculations give photoionization cross-sections, which are several times $(\mathrm{Mg} \mathrm{I})$ to up to 3 orders of magnitude (Fe I) larger than previous, less elaborate approaches. As a result, NLTE calculations lead now to much stronger underpopulation of atomic levels. Varying atomic data, Gehren et al. (2001) have calculated departure coefficients for 236 terms of Fe I in the solar atmosphere. For simple reasons of continuity, one would expect that thermalization of the upper terms with respect to the continuum ground state becomes more complete the smaller the energy difference. The degree of completeness of Fe I levels up to $7.5 \mathrm{eV}$ is very high; however, the upper terms above $6.5 \mathrm{eV}$ do not couple thermally to the Fe II ground state when the data of Bautista (1997) from the Iron Project is used. This indicates that either the role of collisions among highly excited levels is seriously underestimated or photoionization cross-sections of Bautista (1997) are strongly overestimated. In their Fig. 4 Gehren et al. (2001) show that the behaviour of departure coefficients becomes much more reasonable from the physical point of view when $\mathrm{H}$ collisions are included.

How important are inelastic collisions with $\mathrm{H}$ atoms for the kinetic equilibrium of atoms? The basic formula used to calculate collisional rates for allowed transitions is the one originally proposed by Drawin $(1968,1969)$ for colliding atoms of equal mass and later implemented by Steenbock \& Holweger (1984) for the interaction of any heavy atom with $\mathrm{H}$ atoms. Since this approximation provides only an order of magnitude accuracy, the formula published by Steenbock \& Holweger (1984) usually comes with a scaling factor $k_{\mathrm{H}}$. One often cites in the literature the theory treated by Kaulakys $(1985,1986)$, which gives much smaller collisional rates than the Steenbock \& Holweger formula. It should be emphasized that Kaulakys theory can be applied only to transitions between highly excited levels. For Al I Baumüller \& Gehren (1996) and for Mg I Zhao et al. (1998) have found empirically that $\mathrm{H}$ collision cross-sections decrease systematically with excitation energy in rough agreement with Kaulakys's prediction for Rydberg transitions. At the same time NLTE calculations for Fe I (Gehren et al. 2001) favour $\mathrm{H}$ collisional rates about 5 times larger than the values obtained from the Steenbock \& Holweger (1984) formula.

In Table 5, we list empirical determinations of the scaling factor $k_{\mathrm{H}}$, which are mostly based on solar line profile fitting. The only exception is Gratton et al. (1999), where $k_{\mathrm{H}}$ was obtained from a spectral analysis of RR Lyr type stars. Thevenin \& Idiart (1999) found no significant dependence of the distribution of level populations of Fe I on hydrogen collisions; hence, in Table 5 we indicate their result as "no effect". When an atom was investigated in several studies, it can be seen that the obtained values of $k_{\mathrm{H}}$ diverge significantly. This certainly reflects different techniques of NLTE calculations and different model atoms, atomic data and computational details.

And finally, we turn to the problem of collisional rates for forbidden transitions. Such transitions provide coupling of metastable levels to the ground state, coupling of fine structure levels to each other, etc. and play an important role in the kinetic equilibrium of atoms and ions of dominant ionization stages. The theoretical approximation given by Allen (1973) with a collision strength $\Omega=1$ seems to underestimate the electron impact excitation rate (Mashonkina 1996). Some authors use the formula of van Regemorter (1962) adopting $f_{i j}=1 / 10$ 
Table 5. Empirical determination of scaling factor $k_{\mathrm{H}}$

\begin{tabular}{|c|c|c|}
\hline element & $k_{\mathrm{H}}$ & reference \\
\hline $\mathrm{O}$ I & $\begin{array}{l}1 \\
3\end{array}$ & $\begin{array}{l}\text { (Takeda 1995) } \\
\text { (Gratton et al. 1999) }\end{array}$ \\
\hline $\mathrm{Na} \mathrm{I}$ & $\begin{array}{c}0.1 \\
0.05 \\
0.01\end{array}$ & $\begin{array}{l}\text { (Takeda 1995) } \\
\text { (Baumuller et al. 1998) } \\
\text { (Gratton et al. 1999) }\end{array}$ \\
\hline $\mathrm{Mg} \mathrm{I}$ & $\begin{array}{c}1000 \mathrm{e}^{-n E_{n} / 2} \\
3\end{array}$ & $\begin{array}{l}\text { (Zhao et al. 1998) } \\
\text { (Gratton et al. 1999) }\end{array}$ \\
\hline $\mathrm{Fe} I$ & $\begin{array}{c}30 \\
\text { no effect } \\
0.5<k_{\mathrm{H}}<5\end{array}$ & $\begin{array}{l}\text { (Gratton et al. 1999) } \\
\text { (Thevenin \& Idiart 1999) } \\
\text { (Gehren et al. 2001) }\end{array}$ \\
\hline $\mathrm{Sr}$ II & 0.01 & (Mashonkina \& Gehren 2001) \\
\hline Ba II & 0 & (Mashonkina \& Gehren 2000) \\
\hline
\end{tabular}

compared with allowed transitions. Concerning the hydrogen impact excitation rate, there is no theoretical approximation and for most atoms the situation is quite uncertain for transitions between fine structure levels.

With more and more data from quantum mechanical calculations on collisional b-b transitions, it turns out that the approximations cited are even not reliable within an order of magnitude. Quite often the differences amount even to several orders of magnitude.

\section{Conclusion}

The comparison between different NLTE codes lead to a reasonable agreement (better than $2 \%$ in most cases) for LTE level population numbers. However, in NLTE discrepancies between the codes in the line forming regions are still larger, up to $20 \%$ in the Sun and up to $50 \%$ in Vega. This is mainly due to the use of different background opacities (b-b and b-f) in the codes.

We are currently still working on a better agreement for the NLTE level population numbers by implementing the different opacities in the Kiel code. As a conclusion from the comparison of different codes, we strongly suggest a guideline for publication of NLTE calculations. The following information should be given in the paper - possibly in form of electronic tables - to ensure completeness of information

- stellar atmosphere model: $\mathrm{T}_{\mathrm{eff}}, \log g,[\mathrm{M} / \mathrm{H}]$, microturbulence elemental abundances 
- energy levels, statistical weight, parent terms

- lines (b-b), oscillator strength and line broadening parameters

- photoionization (b-f) cross sections

- collisional cross sections (b-b, b-f)

- source of background opacity for b-f and b-b transitions

We hope that this facilitates in the future the comparison between different NLTE studies.

Atomic data used in NLTE calculations have various degrees of accuracy and high accuracy of one type of data does not compensate unsatisfactory accuracy of another type of data. A credence to results of NLTE analyses can only be lent by test calculations and careful checking of the effects of various parameters on the final results.

Acknowledgments. We would like to thank Mats Carlsson, Marc Hempel, Jiri Kubat, and Andreas Korn for stimulating discussions during the conference. ML acknowledges with gratitude the International Astronomical Union for financial support of participation in IAU Symposium No. 210. ML has been partially supported by the Russian Basic Researches Fund (grant 02-02-17174). IK acknowledges travel support by the Leids Kerkhoven-Bosscha Fonds.

\section{References}

Allen, C.W. 1973, Astrophysical Quantities. Athlone Press

Auer, L.H., Heasley, J.N., Milkey, R.W. 1972, Kitt Peak Nat. Obs. Contr. 555

Auer, L.H., Heasely, J.N. 1976, ApJ, 205, 165

Baumüller, D., Butler, K., Gehren, T. 1998, A\&A, 338, 637

Baumüller, D., Gehren, T. 1996, A\&A, 307, 961

Baumüller, D., Gehren, T. 1997, A\&A, 325, 1088

Bautista, M.A. 1997, Astron. Astrophys. Suppl. Ser., 122, 167

Baschek, B., Holweger, H., Traving, G. 1966, Abhandlungen aus der Hamburger Sternwarte, Bd.8, Nr.1

Belyakova, E., Mashonkina, L. 1997, Astron.Zh., 74, 601

Boyarchuk, A.A., Lyubimkov, L.S., Sakhibullin, N.A. 1985, Astrofizika 22, 339

Bruls, J.H., Rutten, R.J., Shchukina, N. 1992, A\&A, 265, 237.

Butler, K., Giddings, J. 1985, Newsletter on Analysis of Astronomical Spectra 9, University of London

Carlsson, M., Judge, P. 1993, ApJ, 402, 344

Carlsson, M., Rutten, R.J., Shchukina, N.G. 1992, A\&A, 253, 567

Carlsson, M., Rutten, R.J., Bruls, J.H.M.J., Shchukina, N.G. 1994, A\&A, 288, 860

Carretta, E., Gratton, R. 1994, Mem. della Soc. Astron.Italiana, 65, 799

De la Reza, R., Torres, C.A.O., Busko, I.C. 1981, MNRAS, 194, 829 
Drake, J. 1991, MNRAS, 251, 369

Drawin, H.-W. 1967, Collision and Transport Cross Sections, Association Euratom-C.E.A., EUR-CEA-FC-383

Drawin, H.-W. 1968, Z.Physik, 211, 404

Drawin, H.-W. 1969, Z.Physik, 225, 483

Gehren, T. 1975, A\&A, 237, 267

Gehren, T., Butler, K., Mashonkina, L., Reetz, J., Shi, J. 2001, A\&A, 366, 981

Giddings, J., 1981, PhD Thesis, University of London

Gigas, D. 1988, A\&A, 192, 264

Gratton, R.G., Carretta, E., Eriksson, K., Gustafsson, B. 1999, A\&A, 350, 955

Holweger, H. 1996, Phys.Scripta, T65, 151

Idiart, T.P., Thevenin, F. 2000, ApJ, 541, 207

Ivanova, D., Shimansky, V. 2000, Astron.Zh., 77, 432

Ivanova, D., Shimansky, V. 2000, Astr.Rep., 44, 376

Kaulakys, B. 1985, J.Phys.B, 18, L167

Kaulakys, B. 1986, Sov.Phys. JETP, 64, 229

Kiselman, D. 1991, A\&A, 245, L9

Kiselman, D. 1994, A\&A, 286, 169

Kiselman, D., Plez, B. 1995, Mem. della Soc. Astron. Italiana, 66, 429

Korotin, S.A., Andrievsky, S.M., Kostynchuk, L.Yu. 1999, Ap\& SS 260, 531

Kurucz, R.L. 1979, ApJS, 40, 1

Kurucz, R.L. 1992, Rev.Mex.Astron.Astrofis., 23, 181

Kurucz, R.L. 1993, Kurucz CD-ROM No. 18. Cambridge, Mass.: Smithsonian Astrophysical Observatory

Lemke, M., Holweger, H. 1987, A\&A, 173, 375

Lemke, M., Venn, K.A. 1996, A\&A, 309, 558

Mashonkina, L. 1996, in Model Atmospheres and Spectrum Synthesis, ASP Conf. Ser., 108, 140

Mashonkina, L., Bikmaev, I.F. 1996, Astron.Zh., 73, 109

Mashonkina, L., Sakhibullin, N., Shimansky, V. 1993, Astron.Zh., 70, 372

Mashonkina, L., Sakhibullin, N., Shimanskaya, N. 1996, Astron.Zh., 73, 212

Mashonkina, L. 2000, Astron.Zh., 77, 630

Mashonkina, L., Gehren, T. 2000, A\&A, 364, 249

Mashonkina, L., Gehren, T. 2001, A\&A, 376, 232

Mashonkina, L., Shavrina, A., Khalack, V., et al. 2002, Astron.Zh., 79, 31

Mishenina, T., Korotin, S., Klochkova, V., Panchuk, V. 2000, A\&A, 353, 978

Paunzen, E., Kamp, I., Iliev, I.Kh., et al. 1999, A\&A, 345, 597

Pavlenko, Ya. 1992, Astron.Zh., 69, 1179

Przybilla, N., Butler, K., Becker, S.R., Kudritzki, R.P., Venn, K.A. 2000, A\&A, 359, 1085

Przybilla, N., Butler, K., Becker, S.R., Kudritzki, R.P. 2001, A\&A, 369, 1009 
Przybilla, N., Butler, K., Kudritzki, R.P. 2001, A\&A, 379, 936

Przybilla, N., Butler, K. 2001, A\&A, 379, 955

Reetz, J. 1999, Ap\&SS, 265, 171

Rentzsch-Holm, I. 1996, A\&A, 312, 966

Rybicki, G.B., Hummer, D.G. 1991, A\&A, 245, 171

Sakhibullin, N.A. 1983, Trudi Kazan gor.obs. 48, 9

Sakhibullin, N.A. 1987, Astron.Zh., 64, 1269

Scharmer, G.B. 1981, ApJ, 249, 720

Shchukina, N. 1987, Kinematika i fizika neb. tel, 3, 40

Shchukina, N., Shcherbina, T. 1990, Kinematika i fizika neb. tel, 6, 44

Shchukina, N.G., Bueno Javier, T., Kostik, R.I. 1997, Solar Physics, 172, 117

Steenbock, W. 1985, in Cool Stars with Excesses of Heavy Elements/ Ed. Jaschek M. and Keenan P. C. Dordrecht: Reidel D., 231

Steenbock, W., Holweger, H. 1984, A\&A, 130, 319

Takada-Hidai, M., Takeda, Y. 1996, Publ. Astron. Soc. Japan, 48, 739

Takeda, Y. 1991, A\&A, 242, 455

Takeda, Y. 1992, Publ. Astron. Soc. Japan, 44, 309.

Takeda, Y. 1992, Publ. Astron. Soc. Japan, 44, 649.

Takeda, Y. 1995, Publ. Astron. Soc. Japan, 47, 463

Takeda, Y., Kato, K., Watanabe, Y., Sadakane, K. 1996, Publ. Astron. Soc. Japan, 48, 511

Thevenin, F., Idiart, T.P. 1999, ApJ, 521, 753

van Regemorter, H. 1962, ApJ, 136, 906

Watanabe, T., Steenbock, W. 1985, A\&A, 149, 21

Zhao, G., Butler, K., Gehren, T. 1998, A\&A, 333, 219. 